\title{
Using Time as a Strategic Element in Continuous Double Auctions
}

\author{
Marcel Neumann, Karl Tuyls, and Michael Kaisers \\ Maastricht University, P.O.Box 616, 6200 MD Maastricht
}

\begin{abstract}
Numerous pricing strategies have been proposed and incorporated into software trading agents. Early simulation experiments have shown that markets are efficient even with only a few traders placing randomly priced offers at each time step using the Zero-Intelligence strategy. This article investigates the strategic effect of timing on the performance of trader profits and market efficiency. The trading strategies Zero-Intelligence and Zero-Intelligence-Plus are enhanced by new timing strategies, replacing their heuristics with random and strategic behavior. As expected, agents make less profit with random timing against the heuristic. However, market efficiency remains the same, confirming that continuous double auctions are highly efficient mechanisms even with traders placing profitable offers with random prices and timing. Furthermore, Zero-Intelligence-Plus agents also achieve high efficiency, but strategic timing reduces the risk of being exploited by trading far from the equilibrium price. Thus, there is a clear individual incentive to exploit timing strategically.
\end{abstract}

Keywords: Continuous Double Auctions, Zero-Intelligence, Timing

\section{Introduction}

Brokers of the New York Stock Exchange (NYSE) exchange about 1.6 billion shares worth $\$ 45$ billion per day. The dominant market mechanism for the exchange of commodity goods is the continuous double auction. Each trader is assumed to have private valuations for the goods to be traded that are only known to itself. Traders place offers at self-elected times to indicate that they are willing to buy (bid) or sell (ask) a good at a certain price. Market clearing occurs each time a new offer arrives. The highest open bid is then tried to be matched with the lowest open ask.

Classic economic theory predicts that the price for one unit of a commodity settles at an equilibrium price where the quantity demanded is equal to the quantity supplied. Smith [8] showed that the transaction price time series has a tendency to converge towards the market equilibrium price even with a relatively small number of human traders. Gode \& Sunder [4] introduced two types of "Zero-Intelligence" computer trading strategies that follow a random pricing scheme. Market efficiency was shown to reach values up to $99 \%$. Cliff 
[1] followed with a more sophisticated trading strategy called "Zero-IntelligencePlus". Traders are able to observe market activity and adjust their offer prices accordingly. Both trading strategies apply the same implicit heuristic about the timing of offers. Offers are placed as soon as their price is being determined.

This article extends both trading strategies Zero-Intelligence and Zero-IntelligencePlus with alternative timing strategies and compares their performance through a series of simulation experiments with their original counterparts. The complexity of the strategies used in this article is summarized in Table 1. The concept of random pricing by Zero-Intelligence agents is transferred and similarly applied to time in order to investigate the performance of agents that do not use time strategically. The complex form of pricing by Zero-Intelligence-Plus agents is enhanced with a sophisticated timing strategy to investigate the performance of agents that use time as a strategic element. This allows to evaluate the performance of traders and markets with varying strategic reasoning about time.

The outline of this paper is as follows. Section 2 introduces the trading strategies to be investigated together with their enhancements and performance measures. Section 3 describes the experiments. It gives a description of the simulation environment and the specific experimental setups together with their results. Finally, the last section draws the conclusions.

\section{Background and Methodology}

\section{Trading Strategies}

Gode \& Sunder [4] introduced two "Zero-Intelligence" (ZI) trading strategies. A trader of the first version submits random offers independently, identically, and uniformly distributed over the range of feasible trading prices from 1 to the maximum trading price enforced by the market mechanism and is referred to as "ZI unconstrained" (ZIU). The second version is subject to a budget constraint that prevents it from engaging in loss making transactions. Thus, buyers submit the uniform random bids between 1 and the valuation of the unit in question. Sellers submit the uniform random asks between the cost of the unit in question and the maximum trading price of the market. It is referred to as "ZI with constraint" (ZIC). Both ZIU and ZIC submit offers as soon as they are computed.

ZI traders are considered to behave randomly. However, the originally proposed "Zero-Intelligence" traders submit offers at every round and thus implicitly use the heuristic always submit. "Zero-Intelligence-Time" (ZIT) traders combine the random pricing of ZI traders with random timing, picking a time $t^{*}$ for the

Table 1. This table lists the sophistication of the strategies' reasoning about the price and time. Each of the strategies is explained in Section 2.

\begin{tabular}{|c|c|c|c|}
\hline Price $\backslash$ Time & Random & Heuristic & Strategic \\
\hline Random & ZIUT & ZIU & \\
Rational & ZICT & ZIC & \\
Heuristic & & ZIP & ZIPT \\
\hline
\end{tabular}


submission of the intended offer, such that $t^{*}$ is distributed uniformly over the anticipated remaining time of the auction.

The trading strategy "Zero-Intelligence-Plus" (ZIP) proposed by Cliff [1] allows agents to observe market activity and adjust future offer prices accordingly. Events in the market that suggest higher profit for a trader result in more profitable offer prices. Similarly, events that show the competitiveness of other traders results in less profitable offer prices. At last, offer prices converge towards unmatched offers in order to stay on a competitive basis. The reader is referred to [1] for a full specification.

"Zero-Intelligence-Plus Time" (ZIPT) enhances the trading strategy of ZIP with strategic timing behavior. The bid-ask-spread is defined by the difference of the price of the lowest open ask and the highest open bid. The size of this spread is used as an indicator of whether prices are going to change in the near future. Offers by ZIPT agents are only submitted when the size of the bid-askspread has fallen below a threshold value. The use of this threshold is inspired by the Kaplan strategy that has won the 1990 Double Auction Tournament despite being among the simplest strategies [7].

\section{Performance Measures}

The profit of a trader $i$ considers all his successful transactions and measures them against the private values $\lambda_{i, j}$ of all units $j$. It is given by

$$
p r_{i}=\sum_{j \in \text { traded }} k\left(\lambda_{i, j}-p_{j}\right),
$$

$k=1$ if $i \in$ Buyers, $k=-1$ if $i \in$ Sellers, where $p_{j}$ denotes the transaction price of unit $j$.

The coefficient of convergence $\alpha$ introduced by Smith [8] measures how close a group of traders trades to the theoretical equilibrium. It is given in equation 1 and it is defined as the standard deviation of the actual trade prices $p_{i}$ from the equilibrium price $p_{0}$ as a percentage of it.

$$
\alpha=\frac{100}{p_{0}} \sqrt{\frac{1}{n} \sum_{i=1}^{n}\left(p_{i}-p_{0}\right)^{2}}
$$

The allocative efficiency $e$ is a measure of performance of an entire market. It is given by equation 2 and defined as the ratio of total actual profit and theoretical profit. Total actual profit is the sum of profits made by each trader while the theoretical profit is the sum of buyers' $s_{b}$ and sellers' $s_{s}$ surplus.

$$
e=\frac{\sum_{i \in \text { traders }} p r_{i}}{s_{s}+s_{b}}
$$

These three measurements give insight into the individual profit, the collective efficiency, and the convergence of the market to equilibrium prices. They provide the basis for the experiments presented in the following section. 


\section{$3 \quad$ Experiments}

The experiments are conducted using a discrete-event simulation. A simulation process runs for six periods. During each period, traders are allowed to submit offers at every time step, and a period is closed after 20 time units without new offers. Traders receive a list of private values at the beginning of each period. The distributions of private values are given in Figures 1, 2, 3 and 4. Offers are for a single unit and subject to the NYSE spread improvement rule. Only one open offer is allowed per trader. A transaction occurs when the price of the highest open bid and the lowest open ask cross each other.

\subsection{ZI vs. ZIT}

A first set of experiments involves homogeneous populations of either ZIU, ZIC, ZIUT and ZICT traders. The population consists of six buyers and sellers re-

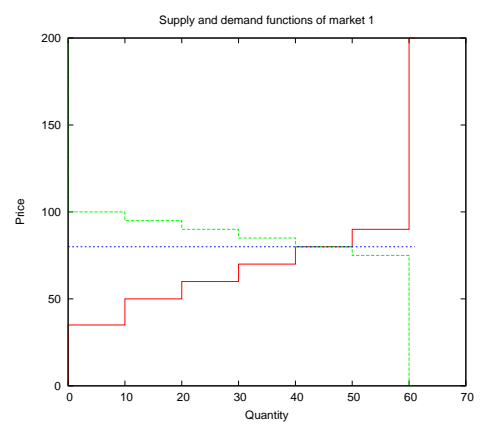

Fig. 1. Supply and demand functions introduced by Gode \& Sunder [4]. Sellers' theoretical profit $=1050$, Buyer's theoretical profit $=500$.

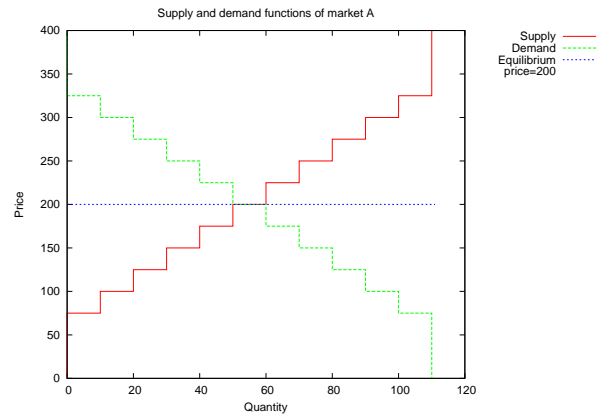

Fig. 3. Supply and demand functions introduced by Cliff [1]. Sellers' theoretical profit $=3750$, Buyers' theoretical profit $=3750$.

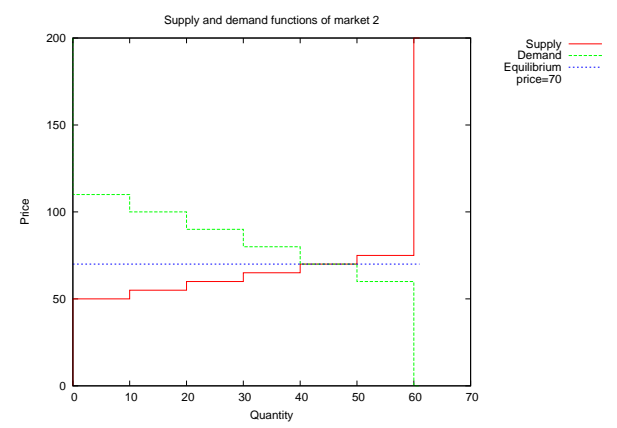

Fig. 2. Supply and demand functions introduced by Gode \& Sunder [4]. Sellers' theoretical profit $=500$, Buyers' theoretical profit $=1000$.

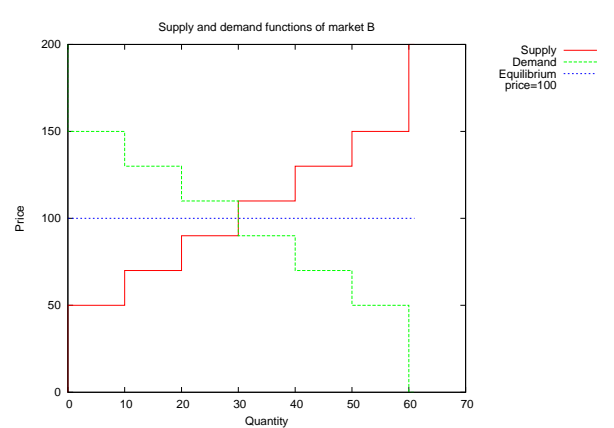

Fig. 4. Seller's theoretical profit $=900$, Buyer's theoretical profit $=900$. 
spectively. The simplification by Gode \& Sunder [4] of dropping all open offers after a transaction is adopted for the sake of comparison.

Gode \& Sunder [4] report that ZIU traders are able to achieve an allocative efficiency of $90 \%$ for market 1 and 2 . ZIC traders are able to raise this value to $99.9 \%$ for market 1 and $99.2 \%$ for market 2 . It is expected that the two types of traders will do equally good in this set-up of experiments. Parsons [5] noted that the efficiency depends on the distribution of private values only. Since ZIUT traders should be able to trade all their units, they should achieve the same efficiency value as ZIU agents. On the other ZICT traders should achieve lower values of allocative efficiency because of a higher risk of extra-marginal units to be traded.

A second set of experiments is concerned with heterogeneous populations of ZI and ZIT traders. ZIU traders are confronted with ZIUT traders while ZIC traders are confronted with ZICT traders. The population consists of twelve buyers and twelve sellers, equally divided into the respective type of traders.

Results Figure 5 shows the transaction price time series in market 1 with a population of ZIU traders. The shape of the graph is identical to the one created by Gode \& Sunder [4]. The pattern can be stipulated as being random. This is also the case for market 2 and $\mathrm{B}$.

The transaction price time series in market 1 with a population of ZIC traders is shown in Figure 6. Again, the shape of the graph is identical to the one created by Gode \& Sunder [4]. The price series is less volatile than the price series of the ZIU traders. Also, the price series converges towards the equilibrium price within each period. The observed pattern is similar for market 2 and B.

Transaction price time series in market 1 with a population of ZIUT traders is shown in Figure 8. The shape of the graph is similar to Figure 5. A random pattern can be observed while periods take longer to finish because traders do

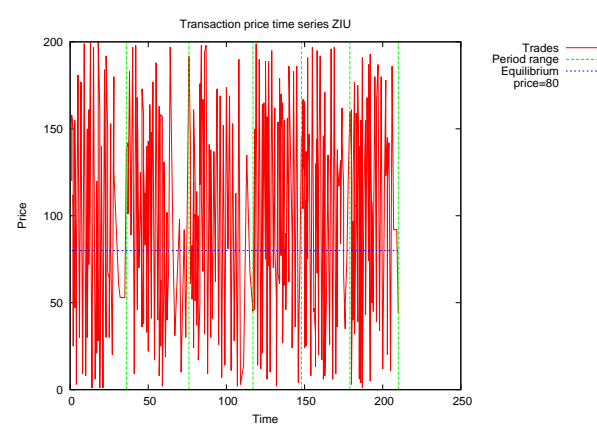

Fig. 5. Transaction price time series for market 1 with 6 buyers and 6 sellers of ZIU traders (heuristic timing).

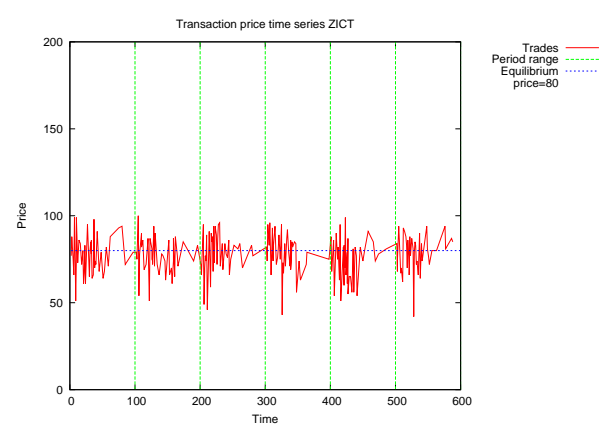

Fig. 6. Transaction price time series for market 1 with 6 buyers and 6 sellers of ZIC traders (heuristic timing). 


\begin{tabular}{|c|c|c|r|}
\hline Traders & $\begin{array}{c}\text { Market 1 } \\
\bar{x}(\mathrm{~s})\end{array}$ & $\begin{array}{c}\text { Market 2 } \\
\bar{x}(\mathrm{~s})\end{array}$ & $\begin{array}{c}\text { Market B } \\
\bar{x}(\mathrm{~s})\end{array}$ \\
\hline ZIU & $90.32( \pm 0.00)$ & $90.00( \pm 0.00)$ & $0.00( \pm 0.00)$ \\
ZIC & $99.17( \pm 0.23)$ & $98.96( \pm 0.27)$ & $97.21( \pm 0.61)$ \\
ZIU-T & $90.32( \pm 0.00)$ & $90.00( \pm 0.00)$ & $0.00( \pm 0.00)$ \\
ZIC-T & $99.12( \pm 0.24)$ & $98.91( \pm 0.28)$ & $96.95( \pm 0.67)$ \\
\hline
\end{tabular}

Fig. 7. Sample mean and sample standard deviation of the efficiency of ZI traders, sample size $=1000$.

not submit offers as soon as possible anymore but randomly distributed over the least remaining time of the period. The pattern is similar for market 2 and $\mathrm{B}$.

Figure 9 shows the transaction price time series in market 1 with a population of ZICT traders. The price series looks similar than the one in Figure 6 . It still converges to the equilibrium price but not as gradually as it was the case for ZIC traders.

Figure 7 shows the sample mean and the sample standard deviation of the allocative efficiency for the experiments above over 1000 runs. The designs of market 1 and 2 imply efficiencies of at least $90 \%$ which occurs when all available units are being traded as it is the case for both types of unconstrained traders. The intra-marginal and the extra-marginal side of the symmetric market B are of equal size. Hence the allocative efficiency of the unconstrained traders in it is 0 . Thus, Gode \& Sunder [4] could have used truly ZIU agents for their experiments already. Introducing a timing heuristic does not increase the efficiency value. In the constrained case however, the introduction of a timing heuristic does lead to a slightly higher efficiency than it is the case with random timing.

The mean actual profits achieved by ZIC and ZICT traders per period in market B when placed in competition is shown in Figure 10. ZIC traders are able to achieve a mean actual profit of above 920 price units while ZICT traders

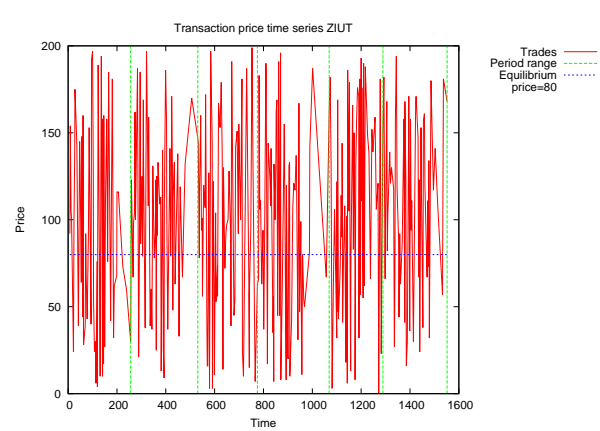

Fig. 8. Transaction price time series for market 1 with 6 buyers and 6 sellers of ZIUT traders (random timing).

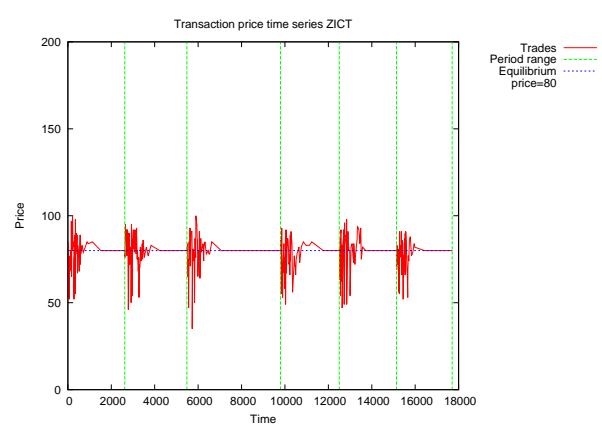

Fig. 9. Transaction price time series for market 1 with 6 buyers and 6 sellers of ZICT traders (random timing). 
are only able to achieve a mean actual profit of around 800 price units. Market theory predicts a theoretical profit of 900 units for this market. This shows that ZIC traders are more competitive due to their heuristic of time. ZICT traders are not able to achieve high profit values. They are even exploited by ZIC traders which explains their profit being above the predicted theoretical profit.

\subsection{ZIP vs ZIPT}

ZIP traders require the following set of parameters. During all experiments $R$ is uniformly distributed over $[1.0,1.05]$ for price increases and over $[0.95,1.0]$ for price decreases. These values are suggested by Cliff [1]. The values for $A$ are changed due to a larger scale for the price units. $A$ is uniformly distributed over $[0.0,5.0]$ for price increases and over $[-5.0,0.0]$ for price decreases. The learning rate $\beta$ is set to 0.3 and the momentum coefficient $\gamma$ is set to 0.05 for all traders as suggested by Preist et al. [6]. At last, buyers start with profit margins that give a price of 0 as their first offer. Sellers on the other hand start with profit margins that give the limit price of the market as their first offer.

ZIPT traders start submitting offers when the size of the bid-ask-spreak has fallen below 5.0 price units. If the bid-ask-spread has not changed for 5 time units ZIPT traders start testing the market themselves before considering the size of the bid-ask-spread again. Also, ZIP traders adjust their profit margins in the direction of the best best open respective offer when there has not been a trade for 5 time units. This policy was introduced by Das et al. [2] and is necessary for ZIP traders to be applied in continuous double auctions.

Unlike the set-up in section 3.1 the list of offers persists after the occurrence of a transaction to provide a more realistic market place.

A first set of experiments is concerned with homogeneous populations of ZIP and ZIPT traders. Cliff [1] reports that transaction prices of ZIP traders converge to the equilibrium price during early periods and stays stable during later periods. The performance of ZIP traders in this set-up should be equally good. Transaction prices of ZIPT traders should also be able to converge to the equilibrium price since they employ the same pricing strategy as their original complement. However, clues for the profit margin adjustments of the traders are given less frequently which might lead to longer period lengths. In a second set of experiments ZIP traders are confronted with ZIPT traders. The performance of either trader is measured by their actual profit earned during any one period.

Results Figure 11 shows the transaction price time series in market A with a population of ZIP traders. As can be seen the transaction prices scallop around the equilibrium price of 200 price units during the first four periods. However, the price series converges towards the equilibrium price where it stays stable for the last two periods.

Figure 13 shows the transaction price time series in market A with a homogeneous population of ZIPT traders. The shape of the graph looks similar to figure 11. The transaction price time series scallops around the equilibrium price for the first four periods and stabilizes in period five. 


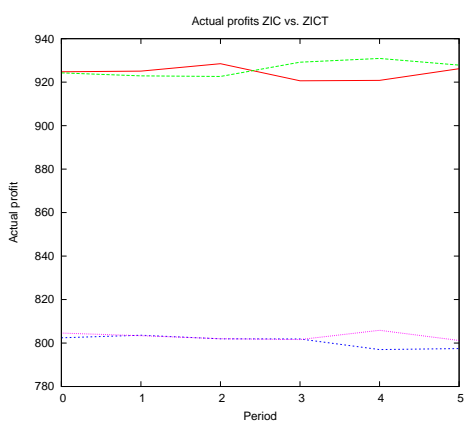

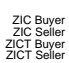

Fig. 10. Actual profits earned in market B by a heterogeneous population of ZIC and ZICT traders.

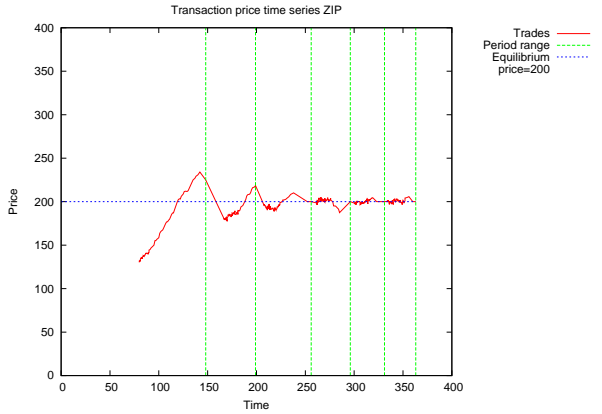

Fig. 11. Transaction price time series for market A with 6 buyers and 6 sellers of ZIP traders (heuristic timing).

\begin{tabular}{|c|c|c|c|}
\hline Traders & Market 1 & Market 2 & Market A \\
& $\bar{x}(\mathrm{~s})$ & $\bar{x}(\mathrm{~s})$ & $\bar{x}(\mathrm{~s})$ \\
\hline ZIP & $99.79( \pm 0.24)$ & $99.86( \pm 0.19)$ & $99.86( \pm 0.30)$ \\
ZIPT & $99.81( \pm 0.22)$ & $99.83( \pm 0.22)$ & $98.84( \pm 0.31)$ \\
\hline
\end{tabular}

Fig. 12. Sample mean and sample standard deviation of the efficiency of ZIP and ZIPT traders, sample size $=1000$.

The mean allocative efficiency of markets with homogeneous populations of either ZIP or ZIPT traders are around $99 \%$ and summarized in figure 12 . The efficiency value for ZIP traders conforms with the findings by Cliff [1].

The second set of experiments was concerned with heterogeneous populations of ZIP and ZIPT traders. Figure 15 shows the transaction price time series while Figure 16 shows the sample mean of the actual profits for each period. It can be

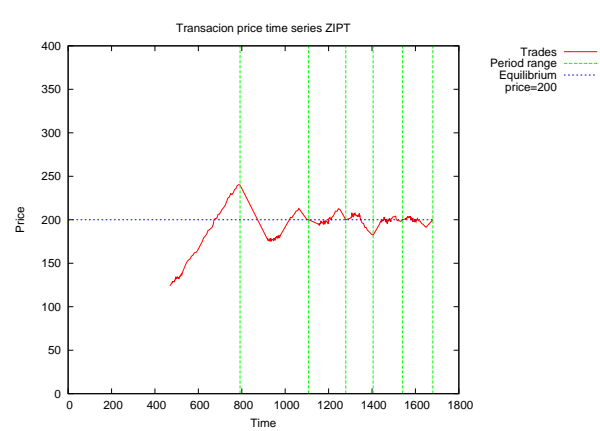

Fig. 13. Transaction price time series for market A with 6 buyers and 6 sellers of ZIPT traders (strategic timing).

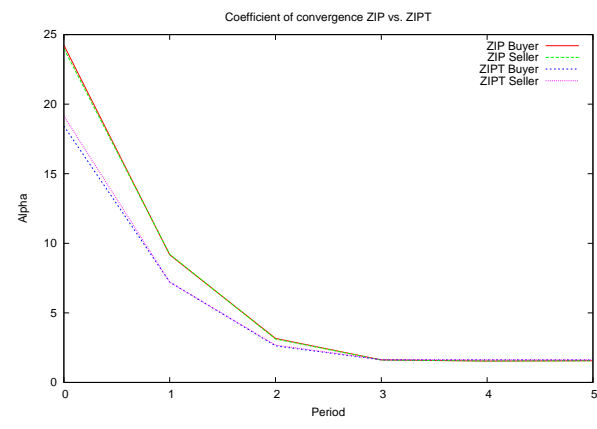

Fig. 14. Course of the sample mean of the coefficient of convergence for heterogeneous populations of ZIP and ZIPT traders, sample size $=1000$. 


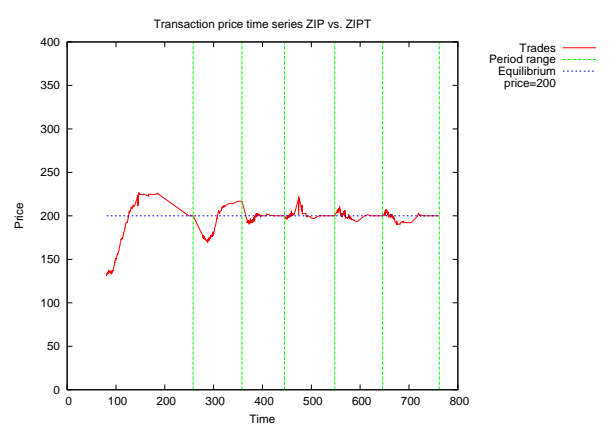

Fig. 15. Transaction price time series for market A with a heterogeneous population of ZIP and ZIPT traders.

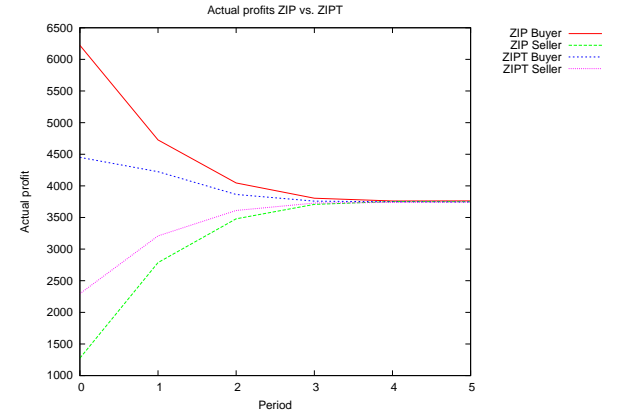

Fig. 16. Course of the sample mean of actual profits for a heterogeneous population of ZIP and ZIPT traders in market A, sample size $=1000$.

seen that ZIPT traders trade closer to the equilibrium price and are therefore less vulnerable to being exploited. It is underlined by Figure 14 which shows the sample mean of the coefficients of convergence for each period.

\section{Conclusions}

This article expanded the horizon of timing strategies employed by trading agents. The implicit heuristic "sooner offers yield higher profits" was made explicit and has been investigated in two directions. First, the Zero-Intelligence trading strategy was enhanced with random timing. The efficiencies of these actual Zero-Intelligence agents does only differ by a very small margin in the case of constrained pricing and it does not differ at all in the case of unconstrained pricing in contrast to their original counterparts. However, when put in competition results of Figure 10 show that using the heuristic yields higher profit than placing random bids in time. This finding confirms that continuous double auctions are highly efficient mechanisms even with only a small number of the most random traders. This efficiency can be expected to increase with the number of traders, but additional experiments are necessary to quantify the scalability and efficiency gains of larger markets.

Second, the Zero-Intelligence-Plus strategy was enhanced with a more sophisticated timing strategy. Again, the results show that the efficiency of the enhancement does not differ from its original complement. In competition to heuristic timing, using time as a strategic element prevented the agents from being exploited by trading far from the equilibrium price, as indicated in Figure 16 .

The outcome of this paper indicate that markets are efficient even with the most random traders, but individual traders do have an incentive to exploit timing as an essential part of their strategies. Intelligent timing strategies are 
indispensable and it can not be made standard practice to only consider timing strategies that place offers as soon as their prices are determined. We suggest to extend this strategic timing to more complex trading strategies such as Gjerstad \& Dickhaut [3], and to discuss the effect explicitly.

\section{References}

1. Dave Cliff. Minimal-intelligence agents for bargaining behaviors in market-based environments. Technical report, School of Cognitive and Computing Sciences, University of Sussex, 1997.

2. Rajarshi Das, James E. Hanson, Jeffrey O. Kephart, and Gerald Tesauro. Agenthuman interactions in the continuous double auction. In Proceedings of the 17th international joint conference on Artificial intelligence - Volume 2, pages 1169-1176, San Francisco, CA, USA, 2001. Morgan Kaufmann Publishers Inc.

3. Steven Gjerstad and John Dickhaut. Price formation in double auctions. GAMES AND ECONOMIC BEHAVIOR, 22:1-29, 1998.

4. Dhananjay K Gode and Shyam Sunder. Allocative efficiency of markets with zerointelligence traders: Market as a partial substitute for individual rationality. Journal of Political Economy, 101(1):119-37, 1993.

5. Simon Parsons, Marek Marcinkiewicz, Jinzhong Niu, and Steve Phelps. Everything you wanted to know about double auctions but were afraid to (bid or) ask. Technical report, in preparation.

6. Chris Preist and Maarten van Tol. Adaptive agents in a persistent shout double auction. In Proceedings of the first international conference on Information and computation economies, ICE '98, pages 11-18, New York, NY, USA, 1998. ACM.

7. John Rust, Richard Palmer, and John H. Miller. Behavior of Trading Automata in a Computerized Double Auction Market. In D. Friedman, J. Geanakoplos, D. Lane, and J. Rust, editors, The Double Auction Market: Institutions, Theories and Evidence. Addison Wesley, 1992.

8. Vernon Smith. An experimental study of competitive market behavior. Journal of Political Economy, 70, 1962. 